\title{
Free-boundary equilibria from stellarator vacuum fields given by their boundary geometry
}

\author{
M.I.Mikhailov ${ }^{1}$, M.Drevlak ${ }^{2}$, S.V.Kasilov ${ }^{3}$, W.Kernbichler ${ }^{4}$, \\ V.V.Nemov ${ }^{3}$, J.Nührenberg ${ }^{2}$, and R.Zille ${ }^{5}$ \\ ${ }^{1}$ National Research Centre "Kurchatov Institute", Moscow 123182, Russia \\ 2 Max-Planck-Institut für Plasmaphysik, Wendelsteinstr. 1, 17491 Greifswald, \\ Germany \\ 3 Institute of Plasma Physics, National Science Center 'Kharkov Institute of \\ Physics and Technology', Akademicheskaya ul. 1, 61108 Kharkov, Ukraine \\ ${ }^{4}$ Institut für Theoretische Physik, Technische Universität Graz, Petersgasse 16, \\ A-8010 Graz, Austria \\ 5 Max-Planck-Institut für Plasmaphysik, Boltzmannstr. 2, 85748 Garching, \\ Germany \\ E-mail: Juergen.Nuehrenberg@ipp.mpg.de
}

Abstract. A stellarator vacuum magnetic field determined by its last closed magnetic surface can be reconstructed by finding a remote surface-current density. Small changes of the surface-current density can then easily be made. These intermediate steps before finding a modular coil system enable free-boundary finite- $\beta$ equilibria to be calculated so that their physical properties can be assessed. 


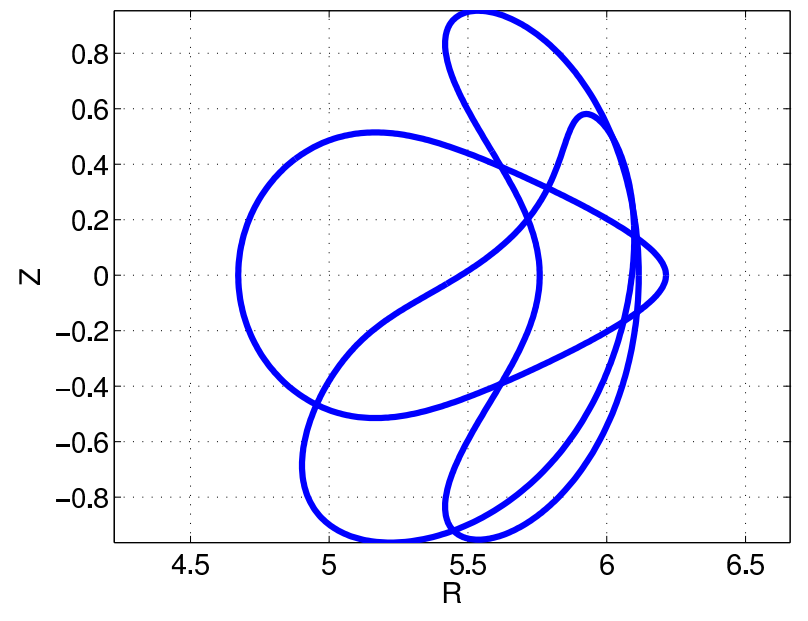

Figure 1. Cuts of the boundary of an optimized configuration [1] at the symmetry planes and in between.

\section{Introduction}

Recently, it has been shown that a non-quasisymmetric stellarator vacuum magnetic field determined by its last closed magnetic surface can exhibit excellent collisionless particle confinement [1]. In [1] a fiveperiod vacuum magnetic field with aspect ratio $A \approx 10$ has been found with a thin $5 / 5$ island chain located at 0.85 of the normalized small radius so that the region of closed magnetic surfaces extends beyond $\iota=1$. Nowadays, a reasonably broad assessment of properties of a magnetic configuration is best done with free-boundary equilibria, in particular, e.g., of its suitability for divertor operation. Traditionally, this has been done by designing a coil system, a procedure often dominated by technical considerations. As an alternative, free-boundary equilibria can be obtained with the help of a moderately remote surface current density so that inappropriate vacuum magnetic fields can be dismissed without coil design. Here this procedure is applied to the stellarator vacuum magnetic field [1] determined by its last closed magnetic surface. The main goals of improvement are to obtain the desired phase of the island chain at the boundary and a reduction of the aspect ratio.

\section{Procedure for free-boundary equilibria}

The optimization in [1] had been done for a W7-Xtype vacuum field (number of periods $\mathrm{N}=5, \frac{5}{6}<\iota<1$ )

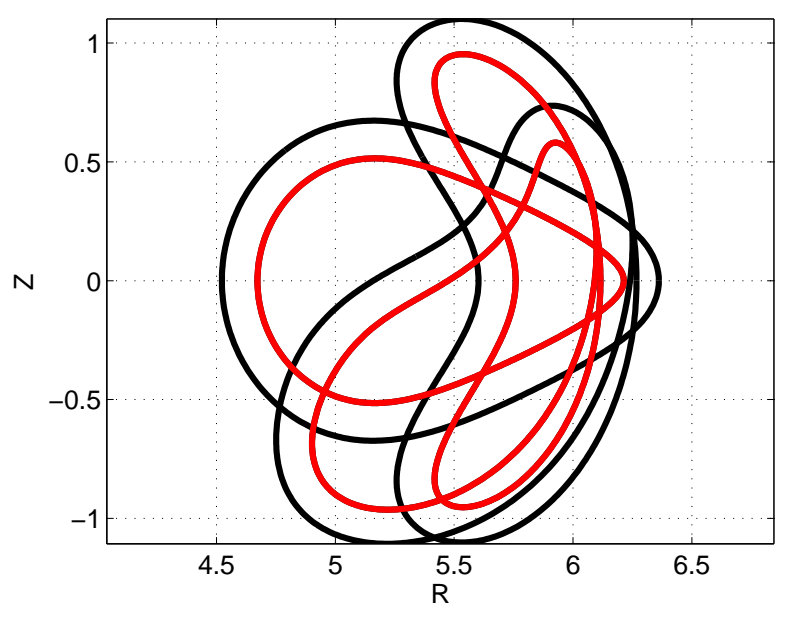

Figure 2. As in Fig. 1 augmented by an outer surface selected to carry the surface current density for reproducing the optimized configuration.

with a boundary at a lower aspect ratio than that of the plasma boundary in order to achieve control of the magnetic field structure in the region intended for divertor operation with a magnetic-island chain at rotational transform unity, see Fig. 1. In Fig. 2, this boundary is seen together with a second surface obtained by equidistant extrapolation.

Then, the surface current density [2] on this second surface is found [3] which reproduces the magnetic field inside the optimized boundary. The surface-current density can be obtained from a surface potential $\Phi[2]$ whose contours are the current density lines. Thus, small changes of the vacuum magnetic field can easily be effected by small changes in $\Phi$. The distance of the current-carrying surface has been chosen such that the contour lines of the surface potential close poloidally and do not incline more than being perpendicular to the poloidal direction. This contour plot is seen in Fig. 3 and the top frame of Fig. 4 shows a Poincaré plot of the reproduction of the original configuration by the surface current density; the original boundary and the corresponding magnetic surface of the reproduction almost coincide, magnetic surfaces even exist beyond this boundary, and the island chain at $\iota=1$ is thin.

Up to this point the vacuum magnetic field created by the surface current density is defined by the boundary of the prescribed vacuum magnetic field. Since the qualitative influence of some of the Fourier coefficients in the surface current potential is obvious, 


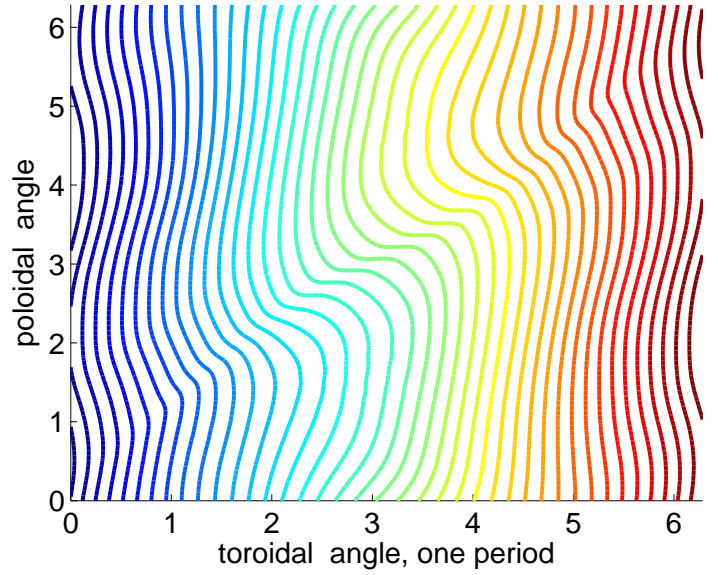

Figure 3. Contour lines of the surface potential on the currentcarrying surface.

promising small adjustments are made before starting free-boundary computations with NEMEC [4]. i) Since it appears possible to decrease the aspect ratio of the confinement region, the longitudinal field (i.e. the secular term proportional to the toroidal coordinate in the surface potential) is increased by $10 \%$ which results in an outward shift of the island chain because of the radially increasing rotational transform. ii) Anticipating the outward plasma shift with increasing plasma- $\beta$, a $25 \%$ adjustment of the Fourier coefficient $\Phi(1,0)$ (roughly corresponding to a vertical field) of the surface potential is made. iii) The original optimization [1] had been performed with the VMEC code so that the phase of the island chain at $\iota=1$ in the vacuum magnetic field is not controlled. Since for a significant width of this island chain the opposite phase as compared to the one seen in the top frame of Fig. 4 appears to be more fitting to the specific triangular shape of the cross-section, a small correction $\left(\mathcal{O}\left(10^{-5}\right)\right.$, but large enough for its purpose) of the Fourier coefficient $\Phi(5,-1)$ is used to change the island phase. These changes are verified to be beneficial by the ensuing free-boundary computations which show that a significantly lower aspect ratio is found than that given by the original surface current density. Without these changes the finite- $\beta$ equilibria exhibit larger aspect ratio and a change of island phase. The bottom frame of Fig. 4 shows the resulting free-boundary equilibrium at very low $\beta$ at the triangular cross-section and Fig. 5 then shows results at the crescent-shaped cross-sectiom of free-boundary calculations with the surface-current density kept fixed: three equilibria at very low, medium and high $\langle\beta\rangle$, with $\langle\beta\rangle$ the average plasma- $\beta$ as given by the NEMEC code. For all $\beta$ values the pressure profile $\mathrm{p} \propto\left(7-11 s+4 s^{2}\right)$ was used and the value of the toroidal flux (which is an input variable of NEMEC) was kept fixed because it turned out that this choice
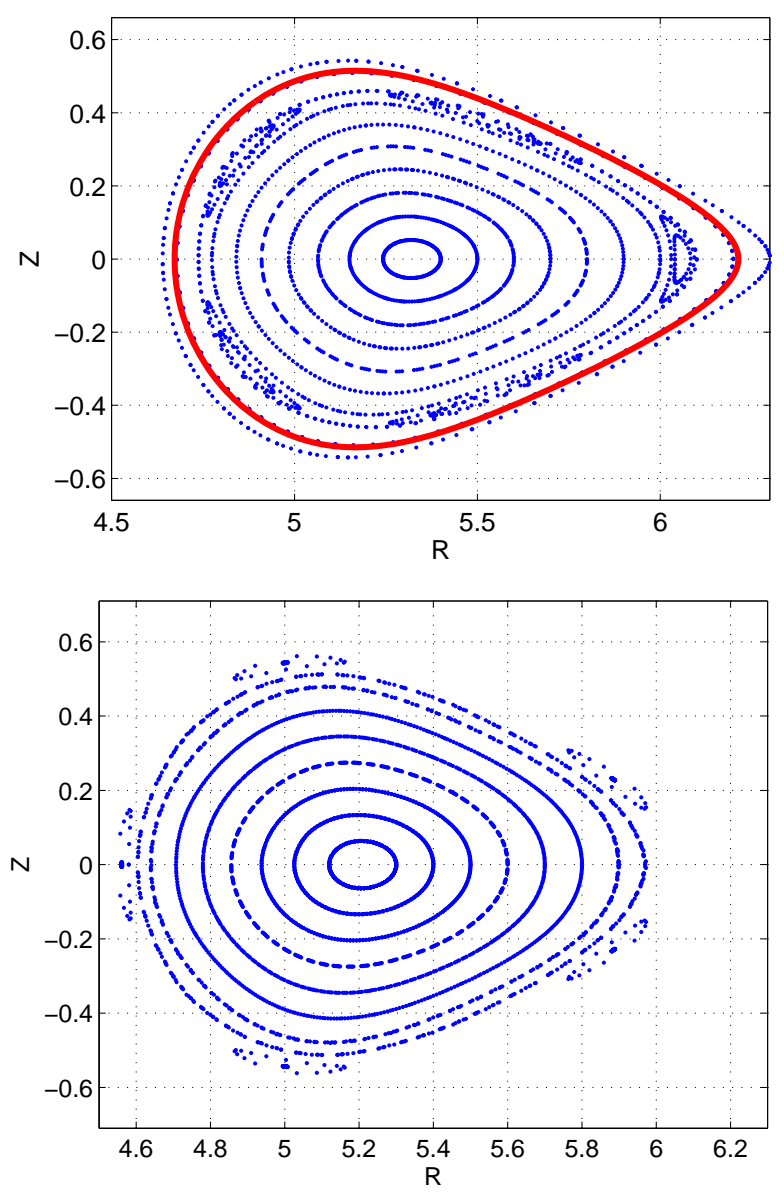

Figure 4. Top: Poincaré plot of the reproduction of the original configuration whose boundary is shown as the continuous line.

Bottom: The very low $\langle\beta\rangle=0.004$, free-boundary equilibrium obtained with the changes of the surface-current density described in the text. As a consequence of the increase of the secular term in the surface potential $\Phi$ the $5 / 5$ island chain is moved to lower aspect ratio, its phase is changed as a consequence of the change of $\Phi(5,-1)$ and the magnetic axis is slightly shifted towards the torus centre as a consequence of the change of $\Phi(1,0)$. For uniformity of representation, here and in Fig. 5, field line following is used inside and outside the plasma boundary; inside, the field lines just represent the NEMEC flux surfaces.

was compatible with the structure of the magnetic field beyond the boundary. The finite- $\beta$ effect on the structure of the magnetic field appears to be sufficiently benign, in particular because the phase of the island chain does not change and the shifts of the o-points with $\beta$ in the crescent-shaped cross-section are small.

\section{MHD-stability and drift-kinetic properties}

As far as MHD-stability properties are concerned the Mercier and resistive interchange (indicative of peeling instability for vanishing net toroidal current) criteria have been checked and found to be stable except for the 

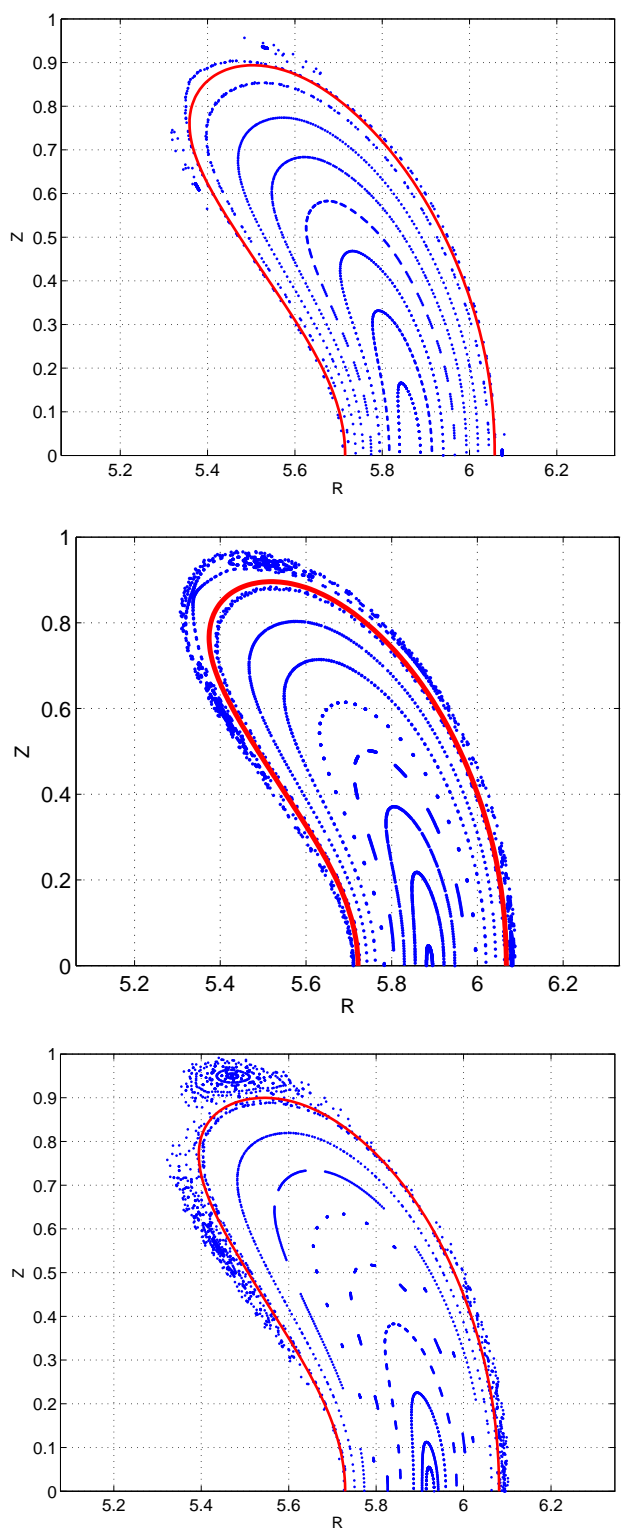

Figure 5. Poincaré plots in the symmetry plane of the crescent cross-section for $\langle\beta\rangle=0.004$ (the same equilibrium as in Fig. 4 bottom), 0.025, 0.05. The continuous lines show the NEMEC boundaries. At the bottom, the seventh flux-surface (counted from the plasma center) is near $\iota=10 / 11$.

resonance at $\iota=10 / 11$ whose relevance was discussed, e.g. [5] .

As to drift-kinetic properties, the neoclassical ripple $\epsilon^{\frac{3}{2}}$, the structural factor $G_{b}$ characterizing the bootstrap current and fast-particle losses were computed as in earlier work (see, e.g. [6] and references cited therein). These results are seen in Fig. 6 to Fig. 8 and show that they are sufficiently small, in case of the energetic-particle loss however only at finite $\beta$. The poor collisionless confinement at low $\beta$ is caused by a transition in the structure of the contours of the second adiabatic invariant $\mathcal{J}$ : while

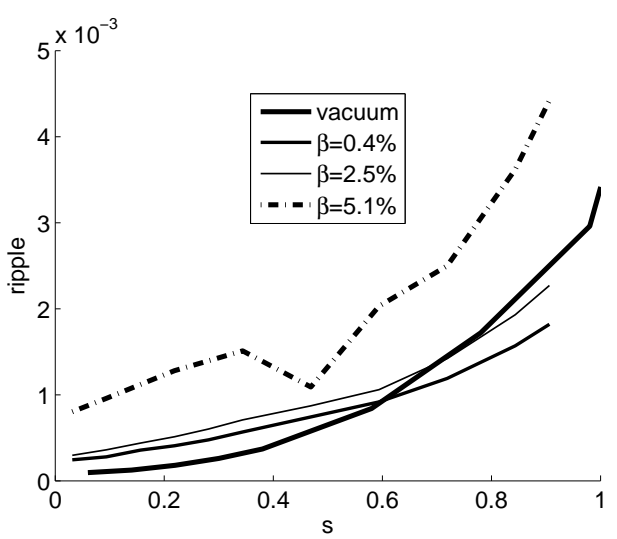

Figure 6. Profiles of the equivalent ripple (the $3 / 2$ power of it). The pecularity of the radial behavior at high $\beta$ is attributed to the elimination of contours of the second adiabatic invariant leading from the plasma core to its boundary across the flux surface at which the ripple is evaluated.

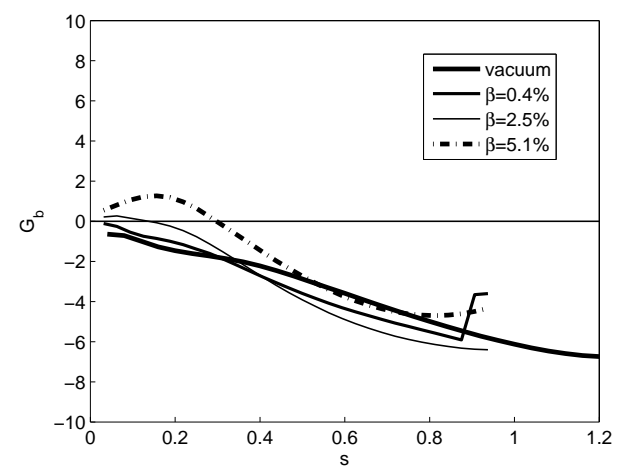

Figure 7. Profiles of the structural factor of the bootstrap current. The abrupt change for $\langle\beta\rangle \approx 0.004$ near the boundary is caused by a change in the position of $B_{\max }$ where the boundary condition for the evaluation of $G_{b}$ is applied.

at $\beta=0$ a mixture between a minimum- $\mathcal{J}$ for deeply reflected particles and a maximum- $\mathcal{J}$ situation for barely reflected particles exists, a pure maximum- $\mathcal{J}$ situation is established at medium $\beta$.

\section{Conclusion}

By extending the region of optimization beyond the plasma boundary [1] and employing a surface current density remote from the plasma and divertor regions, as done here, a stellarator vacuum field with the confinement region aspect ratio $A \approx 10$ has been found whose free-boundary equilibria exhibit properties attractive for fusion application.

\section{Acknowledgments}

This work was supported in part by the Russian Federation Presidential Program for State Support of 


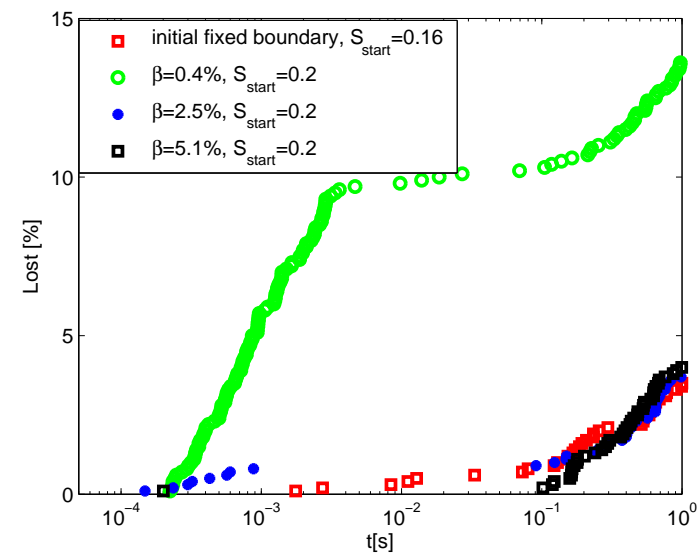

Figure 8. Loss histories of 1000 collisionless $\alpha$-particles evaluated in a fusion-type device $\left(B=5 \mathrm{~T}, \mathrm{~V}=10^{3} \mathrm{~m}^{3}\right)$. Each symbol represents the loss of one particle. The red squares repeat the result of Ref. 1. No particle is lost for $\langle\beta\rangle=0.025$ between 0.001 and $0.1 \mathrm{sec}$ and for $\langle\beta\rangle=0.051$ only one particle is lost before $0.1 \mathrm{sec}$. The $\mathrm{s}_{\text {start }}$ values indicate the starting flux surfaces with $\mathrm{s}$ the normalized toroidal flux $(0<\mathrm{s}<1)$.

Leading Scientific Schools (grant no. NSh-3328.2014.2). This work has been carried out within the framework of the EUROfusion Consortium and has received funding from the Euratom research and training programme 2014-2018 under grant agreement No 633053. The views and opinions expressed herein do not necessarily reflect those of the European Commission. The support of Prof. P. Helander is gratefully acknowledged.

\section{References}

[1] Kasilov S V, Kernbichler W, Mikhailov M I, Nemov V V, Nührenberg J and Zille R 2013 Plasma Physics Reports 39 334-336

[2] Merkel P 1987 Nuclear Fusion 27867

[3] Merkel P and Drevlak M 1998 ICPP and 25th EPS Conf. on Contr. Fus. and Plasma Phys., Prague 1998 (Europ. Conf. Abstr. vol 22C) (Geneva: Europ. Phys. Soc.) p 1745

[4] Hirshman S, van Rij W and Merkel P 1986 Comp. Phys. Comm. 43143

[5] Mikhailov M I, Nührenberg J, Strumberger E and Zille R 2011 Plasma Physics Reports 37 391-394

[6] Subbotin A, Mikhailov M, Shafranov V, Isaev M, Nührenberg C, Nührenberg J, Zille R, Nemov V, Kasilov S, Kalyuzhnyj V and Cooper W 2006 Nuclear Fusion 46 921 